\title{
Plasmacyte-reticulum cell satellitism in multiple myeloma associated with amyloidosis
}

\author{
T S Pillay, G Sayers, A R Bird, P Jacobs
}

\begin{abstract}
A novel morphological feature is described in a patient with myeloma and associated amyloidosis: characteristic clustering (satellitism) of neoplastic plasma cells around macrophages in bone marrow aspirates. Although described in myeloma cell culture, as far as is known, this is the first description of this phenomenon in a patient. This unique association may partly explain the origin of amyloid deposition in tissues and organs.
\end{abstract}

Durie et al provided morphological evidence for amyloid secretion by macrophages that
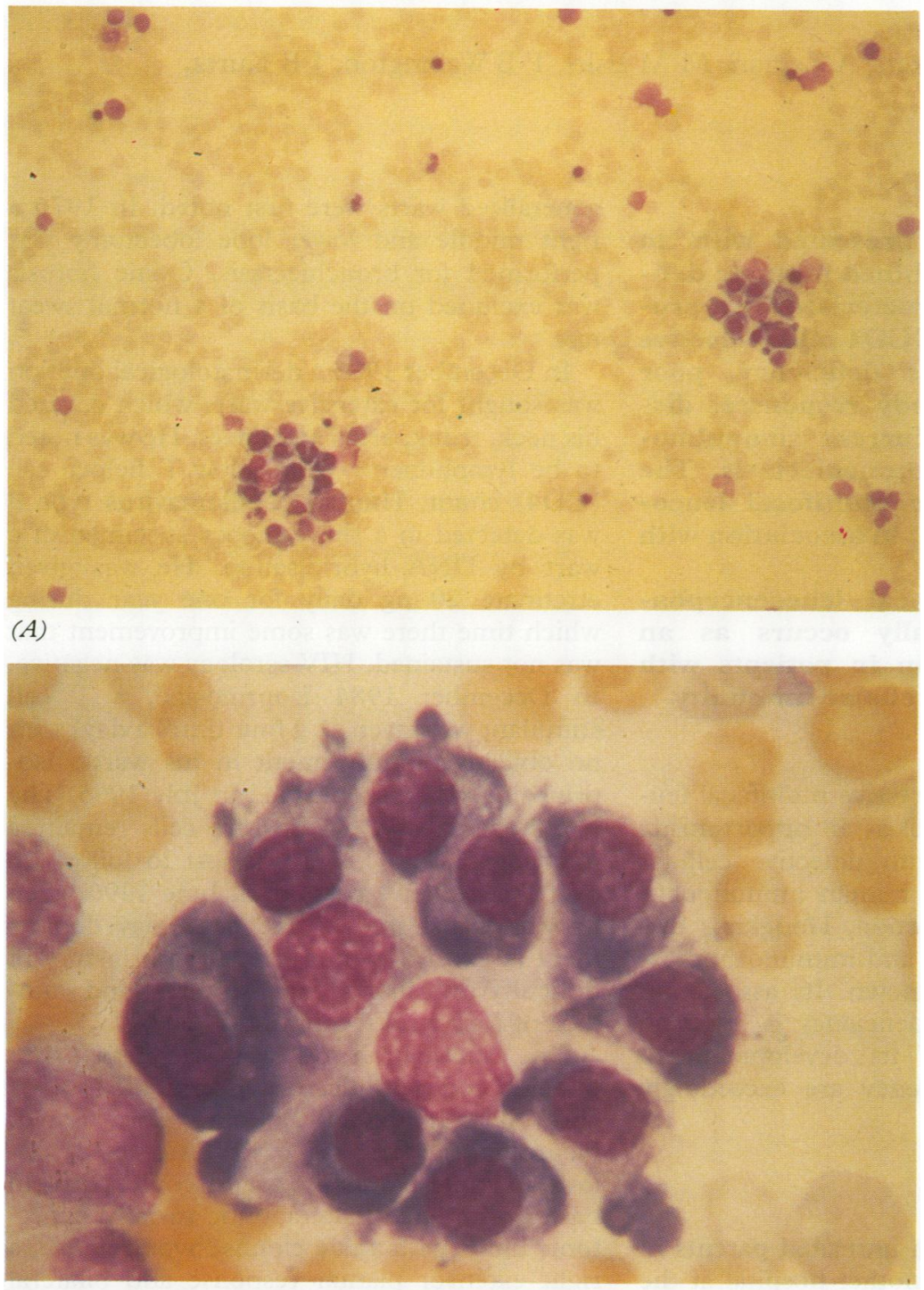

(B)

Figure (A) Bone marrow aspirate smear. Groups of plasma cells are seen clustered around reticulum cells. (B) High power view of a plasma cell cluster with a reticulum cell
nucleus visible in the centre. were present in human myeloma stem cell cultures. ${ }^{1}$ We report an analogous phenomenon observed in the bone marrow aspirates of one of our patients with myeloma and associated amyloidosis that lends support to this in vitro finding in vivo.

\section{Case report}

A 78 year old man of varied ancestry had multiple myeloma that had been diagnosed six years ago on the basis of a bone marrow biopsy specimen and the presence of an $\operatorname{IgG} \lambda$ monoclonal gammopathy $(17 \mathrm{~g} / \mathrm{l})$ with immune paresis and osteolytic lesions. During the course of follow up he developed hepatomegaly and macroglossia with AL type amyloidosis which was confirmed in rectal and tongue biopsy specimens.

Owing to the presence of severe osteoporosis and vertebral collapse which distorted surface landmarks a bone marrow trephine biopsy was technically impossible and only a bone marrow aspirate was obtained. Two aspirates, taken a year apart and stained by the May-GrünwaldGiemsa technique, showed numerous dysplastic plasma cells, some with characteristic Russell bodies, accounting for between $60-70 \%$ of the nucleated cells in the preparation. A distinctive feature was the plasmacytic cuffing around macrophages (figure).

\section{Discussion}

Amyloidosis occurs in about $15 \%$ of patients with myeloma. ${ }^{23} \mathrm{~A}$ pathogenetic mechanism has been ascribed to the secretion of amyloid protein by macrophages present among the myeloma cells, based on findings derived from myeloma stem cell cultures. ${ }^{145}$ A close association was noted between the tumour cells and macrophages and the presence of well orientated tufts of amyloid fibrils shown at the invagination sites beside the packed lysosomes, suggesting that this was predominantly a secretory process by the reticuloendothelial cells. ${ }^{1}$ This concept of amyloidogenesis has, however, been challenged by other workers. ${ }^{6}$

The distinctive morphological features (figure) are essentially similar to those reported in culture by Durie and coworkers, ${ }^{1}$ although these authors stated that this feature could not be demonstrated in the bone marrow examinations carried out on their patient. The plasmacytic satellitism in our patient was noted on two separate occasions, one year apart, thereby excluding any artefactual cause for this finding. Because our patient had histologically confirmed amyloidosis, we suggest that the present finding represents the in vivo equiva- 
lent of that observed in culture, and consequently macrophages may be centrally involved in amyloid production. If that is correct, cells of the reticuloendothelial system may become targets for treatment in patients with myeloma.

Support was provided by the University of Cape Town Leukaemia Centre and Staff Research (Cancer) Fund, the National Cancer Institute, the Medical Research Council, the Michael Chanani and Kaliski Bequests and the Gwendoline Moore Trust.
1 Durie BGM, Persky BP, Soehnlen BJ, Grogan TM, Salmon $\mathrm{SE}$, Amyloid production in human myeloma stem-cell culture, with morphologic evidence of amyloid secretion by associated macrophages. $N \mathrm{Engl} f \mathrm{Med} 1982$; 307:1689-92.

2 Glenner GG, Page DL. Amyloid, amyloidosis and amyloidogenesis. Int Rev Exp Pathol 1976;15:1-92.

3 Kyle RA, Bayrd ED. "Primary" systemic amyloidosis and myle RA, Bayrd ED. "Primary" systemic amyloidosis and
myeloma. Discussion of relationship and review of 81 myeloma. Discussion of relationship and

4 Kjeldsberg CR, Eyre HJ, Totzke H. Evidence for intracellular amyloid formation in myeloma. Blood 1977;50:492-504

5 Hopper KE, Wood PR, Nelson DS. Macrophage heterogeneity. Vox Sang 1979;36:257-74.

6 Zucker-Franklin D, Frangione B. Amyloid in myeloma stem-cell culture. $N$ Engl $₹$ Med 1983;308:1164-5.

\title{
Progressive multifocal leucoencephalopathy, sclerosing cholangitis, bronchiectasis and disseminated warts in a patient with primary combined immune deficiency
}

\author{
S A Misbah, G P Spickett, A Zeman, M M Esiri, T B Wallington, J B Kurtz, \\ H M Chapel
}

\begin{abstract}
A 24 year old man presented with an unusual primary combined immune deficiency syndrome characterised by a profound lymphopenia of CD4 cells, selective serum IgG2 subclass deficiency, poor polysaccharide antibody responses, disseminated warts, recurrent sinopulmonary infection and bronchiectasis. The developed progressive multifocal leucoencephalopathy (PML) in association with sclerosing cholangitis.
\end{abstract}

Progressive multifocal leucoencephalopathy (PML) usually occurs as an opportunistic infection in patients with secondary defects in cellular immunity.

Department o

S A Misbah

G P Spickett

H M Chapel

Department of

Neurology

A Zeman

Department of

Neuropathology

M M Esiri

Department of

Virology

J B Kurtz

John Radcliffe

Hospital and Radcliffe

Infirmary, Oxford

Department of

Immunology,

Southwestern Blood

Transfusion Service,

Bristol

T B Wallington

Correspondence to:

Dr S Misbah, Departmen

of Immunology, Level 7

John Radcliffe Hospital,

Oxford OX3 9DU

Accepted for publication

19 November 1991
A caucasian boy born to unrelated parents in 1966 was first seen at another hospital at the age of 3 years on account of flexural eczema, recurrent tonsillitis, and otitis media. By the age of 5 a hoarse voice led to the detection and removal of vocal cord polyps. Two years later generalised warts were first noted. In 1976 a right middle and lower lobe lobectomy was performed for bronchiectasis. Cystic fibrosis was excluded on the basis of a normal sweat test.

In December 1983 a dermatological opinion was sought for extensive warts which covered his neck, trunk, arms and knees. He was noted to be lymphopenic with a low $\mathrm{T}$ helper cell (CD4) count. Human papillomavirus type 3 was detected in a shave biopsy specimen of a wart by DNA hybridisation. He was given etretinate $20 \mathrm{mg}$ daily for one year during which time there was some improvement that was not sustained. HIV serology was negative. In December 1984 Isoprinosine, a $\mathrm{T}$ cell stimulant was given ( $1 \mathrm{~g}$ four times a day) with no objective improvement in his warts. Isoprinosine was discontinued in July 1985. The lymphopenia of CD4 positive cells remained unchanged (fig 1). He was lost to follow up over the next five years until June 1990 when he was transferred to the neurology department at Oxford with a three month history of difficulty in walking, focal seizures, and jaundice of four weeks' duration.

On examination he was cachectic, dyspnoeic, and jaundiced. Disseminated warts and bilateral digital clubbing were noted. Neurologically he was partly orientated, followed simple commands, and could perform basic arithmetic. Bilateral optic atrophy, hypertonic limbs with ankle clonus, hyperreflexia, a right extensor plantar response and bilateral cerebellar deficits were noted. A cranial computed tomogram showed a left cerebellar hemispheric cyst with numerous low density lesions in both thalamic nuclei, right caudate 